\title{
Verbreitung von Ambrosia (Götterspeise) und das damit verbundene Gesundheitsrisiko: Wird die Schweiz dieser Invasion standhalten?
}

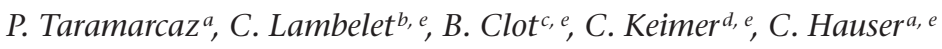

a Service d'Allergologie et Immunologie, Hôpital Universitaire de Genève

b Conservatoire et Jardin botaniques de la Ville de Genève

c MétéoSuisse, Biométéorologie et environnement, Station aérologique, Payerne

d Service de l'agriculture, Station phytosanitaire, Genève

e Genfer Ambrosia-Gruppe

Korrespondenz:

Prof. Dr. med. Conrad Hauser

Service d'Allergologie et Immunologie Hôpital Universitaire de Genève

24, rue Micheli-du-Crest

CH-1211 Genève 14

E-Mail:

Conrad.Hauser@medecine.unige.ch
Diese Seite soll die Ärzteschaft über die Gefahr orientieren, welche vom Unkraut Ambrosia (Götterspeise, Traubenkraut, Ambrosia artemisiifolia) für Umwelt und Gesundheit ausgeht. Die Seite wurde von Experten in Botanik, Pollenwissenschaft, Pflanzenökologie, Agronomie und Allergie verfasst.

Gebiete in Europa, welche stark mit Ambrosia kolonisiert sind, umfassen das Rhonetal, Norditalien, Österreich, Ungarn, Bulgarien und einige Staaten des ehemaligen Jugoslawien. In diesen Gebieten leiden bis zu 12\% der Bevölkerung an Allergien (allergische Rhinokonjunktivitis, Asthma), welche von Ambrosiapollen hervorgerufen werden. Die Schweiz hat begonnen, von dieser Pflanze kolonisiert zu werden. Ambrosiapollenmengen in der Luft werden an verschiedenen Punkten der Schweiz gemessen. Bislang sind die Konzentrationen niedrig geblieben, können aber lokal Spitzen erreichen, welche bei allergischen Individuen bereits Symptome hervorrufen. Trotzdem ist Ambrosiapollenallergie heute noch selten in der Schweiz. Weil die Menge an Ambrosiapollen in den letzten paar Jahren zugenommen hat, wurde mit der Identifikation und Überwachung von Ambrosiawuchs begonnen. Im Kanton Genf und im Südtessin wird Ambrosiawuchs systematisch erfasst. Eine Massierung von Ambrosiawuchs wurde im Kanton Genf, dem westlichen Genferseeufer des Kantons Waadt und im Südtessin registriert. Hauptsächliche Invasionsrouten stellen Autobahnen und Bahnlinien dar. Einzelherde könnten durch kontaminiertes Vogelfutter oder Saatgut verursacht sein. Informationen zur Identifikation und Bekämpfung werden in Genf von staatlichen Instanzen abgegeben. Staatliche Instanzen befassen sich auch mit der Bekämpfung von Ambrosia auf öffentlichem Grund. Neue Methoden der Ausmerzung werden z.Z. von verschiedenen Stellen studiert. Die Dringlichkeit einer wirksamen Bekämpfung der derzeitig noch geringen Ambrosiadurchseuchung erklärt sich durch die praktische Unmöglichkeit, dieses Unkraut in schwer kolonisierten Gebieten auszurotten. Es wird vorgeschlagen, dass Bundesinstanzen die Verbreitung von Informationen über Ambrosia und deren Prävention koordinieren. Bundesinstanzen sollten sich auch bei der Abfassung von Empfehlungen über die Methoden der Ambrosiaausbreitung beteiligen. Schliesslich soll die Bundesgesetzgebung eine gesetzliche Basis einer Ambrosiaprävention schaffen, z. B. durch Kontrolle von importiertem Vogelfutter und Saatgut. Die Kosten einer Prävention der Ausbreitung von Ambrosia sind sicherlich um Grössenordnungen niedriger als die Behandlung von Ambrosiapollenallergie bei einem signifikanten Prozentsatz der Bevölkerung (z. B. könnten die Kosten 100 Mio. Schweizer Franken pro Jahr pro 100000 Ambrosiaallergiker erreichen). Weil sich eine Intensivierung der Ambrosiakolonisierung in wenigen Jahren entwickeln kann, sollte die vorliegende zeitlich limitierte Gelegenheit zur Eindämmung von Ambrosia in der Schweiz nicht verpasst werden.

Eine detailliertere Übersichtsarbeit zu diesem Thema von derselben Autorengruppe wird demnächst im Swiss Medical Weekly erscheinen. Der Zugang zur elektronischen Version ist gratis (www.smw.ch). 